\title{
Experimental Study and CFD Simulation of Pool Fires
}

\author{
Alireza Alizadeh Attar \\ Corresponding Author \\ Petroleum University of \\ Technology
}

\author{
Mojtaba Pourmahdian \\ MSc student of Petroleum \\ University of Technology
}

\author{
Bagher Anvaripour \\ Petroleum University of \\ Technology
}

\begin{abstract}
Liquid fuels(hydrocarbon and peroxide)are flammableand there is a risk of pool fires during their storage and transportation. In order to measure and develop effective methods for protection and considering safety distances, experimental studies and CFD simulations of pool fire of two hydrocarbon fuels (i.e. gasoline and kerosene) and peroxide (TBPB (tert-butyl peroxybenzoate)) were performed. Experiments revealed that the pool fires of liquid organic peroxide show fundamentally very different characteristics e.g. generally much higher mass burning rate, largerflame length as well as higher temperature and subsequently higher irradiance in comparison to liquid hydrocarbon pool fires. The three well-known flame zones of pool fires is well captured by CFD simulations and the predicted axial flame temperature profiles.The safety distances accurately predicted by CFD simulations when predicted time averaged maximum flame temperature is used instead to the experimentally measured values in calculations performed in this work.
\end{abstract}

\section{Keywords}

CFD simulation,pool fire,temperature, irradiance

\section{INTRODUCTION}

Pool fire is the most common consequence of any accidentally released flammable liquid. The occurrence of such scenario may appear in a storage facility or during the transportation of dangerous goods. A number of measures have been developed for specifying the safety distances from such fires for the people and places. The characteristics of large pool fire e.g. burning rate, flame length and radiation are the most common parameters of interest. There have a lot experimental work [2] been done on the measurement of these characteristics. Recently some computational work has also been reported by various groups [4]. The prediction of flame temperature,velocity, irradiance and surface emissive power were carried out by using CFD simulation with various submodels for turbulence and chemistry. The decision about the distance for their safe storage and transportation is made on the basis of standard fire tests. Burning rate, flame temperature and irradiance are the important parameters of concern.

\section{DYNAMICS OF POOL FIRES}

The structure of most pool fires may be split into a number of fairly well-defined zones [1,3,5-7]. These zones are shown in "Figure 1"[7] and the physical processes along with their technical importance is described below.

The liquid fuel itself : In deep pools there may be a significant convective flow within the fuel which can affect the fuel vaporization rate.

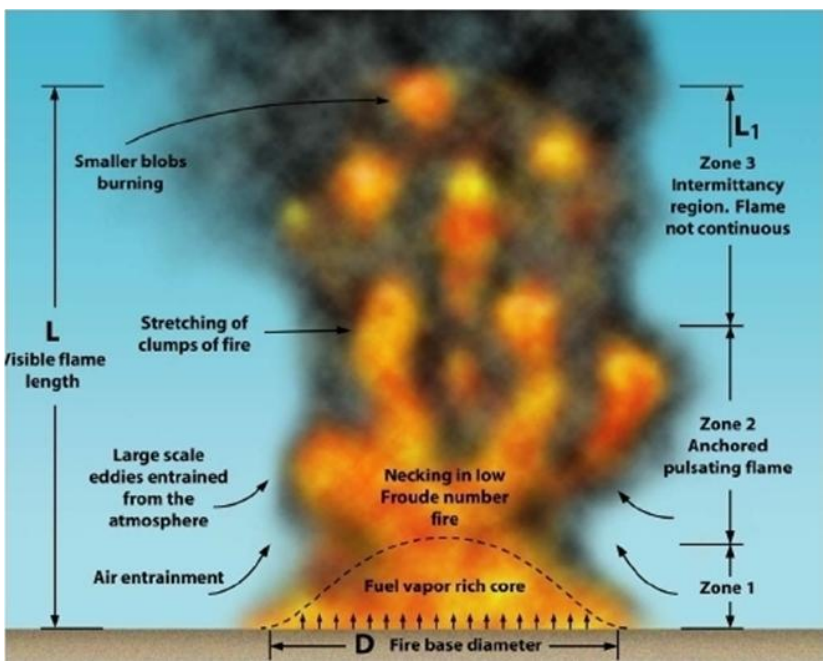

Fig 1: Existence of three zones in a turbulent buoyant diffusion flame

A zone of un-burnt fuel vapor above the liquid fuel, which is usually close to a constant conical shape.

A combustion region above this zone but here there is intermittency and obvious turbulence in the reactive flow.

The non-reacting buoyant plume which is generally fully turbulent in nature and is characterized by decreasing velocity and temperature with height and lateral position.

Physical characteristics associated with the pool fire have a direct impact on the different zones and this impact is generally defined by means of measurable quantities.

\section{EXPERIMENTAL PROCEDURE}

The arrangement of tests is shown in "Figure 2" where the fuel pan was placed on top of a weighing instrument. The thermocouples were located at increased axial distance above the liquid pool surface. The video camera is located at a defined location (depending on the intensity of the flame) from the flame. The fire experiments from pool diameter of $0.06 \mathrm{~m}$ to $0.18 \mathrm{~m}$ were carried out in the laboratory. The size and the materials of the pans are given in "Table 1".

Flame lengths were measured by using the movie sequence recorded by a video camera. The instantaneous images of the flame obtained during the main burning period were then converted into time averaged value of the flame length. 


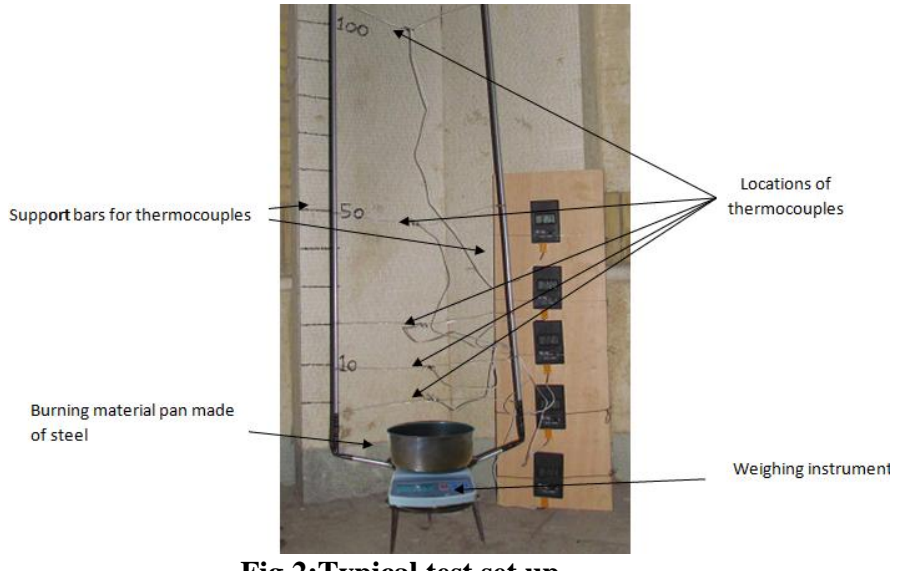

Fig 2:Typical test set up

Table 1. Table captions should be placed above the table

\begin{tabular}{cccc}
\hline Pan material & $\begin{array}{c}\text { Pool } \\
\text { diameter } \\
(\mathbf{m})\end{array}$ & Pan Height(m) & Pan wall(m) \\
\hline steel & 0.06 & 0.05 & 0.002 \\
steel & 0.11 & 0.05 & 0.002 \\
steel & 0.18 & 0.05 & 0.002 \\
\hline
\end{tabular}

\section{MODELING AND SIMULATION}

\subsection{Procedure of CFD simulation}

In present work, pool fires of different hydrocarbons are simulated. The CFD simulation is done by using different submodels contained in softwares ANSYS FLUENT. The different pool diameters are used to show that the experimentally found dependence of various parameters such as temperature and thermal radiation on pool diameter in the calculations realistically reproduced the experimental data. The main purpose of CFD simulation was to determine the temperature T, Surface Emissive Power (SEP) and irradiance E. In the present CFD simulations, turbulence modeled using RSM turbulence model with a buoyancy correction term and Large Eddy Simulation (LES). Due to the absence of detail of the combustion reaction of different fuels studied in this work, a single step reaction and eddy-dissipation model used for combustion modeling. Soot formation is modeled using onestep Khan and Greeves model and the Discrete Ordinate (DO) radiation model is applied for thermal radiation modeling which is the most detailed radiation model applied in AnsysFLUENT.

\subsection{Geometry,mesh and boundary condition}

In this work three-dimensional (3-D) simulation of pool fires has been carried out. The domain is modeled as a cylindrical shape surrounded the pool of liquid fuel. The cylindrical domain discretized to the hexahedral non uniform structured control volumes. The details of the mesh and boundary types are given in "Figure 3". The mesh is very refined at the pool surface and in the inner part of domain with increasing cell dimension as moving to the side boundaries. The number of the meshes varied according to the size of the pool. The meshes and geometries which are used in the present simulations are created using Ansys-ICEM software

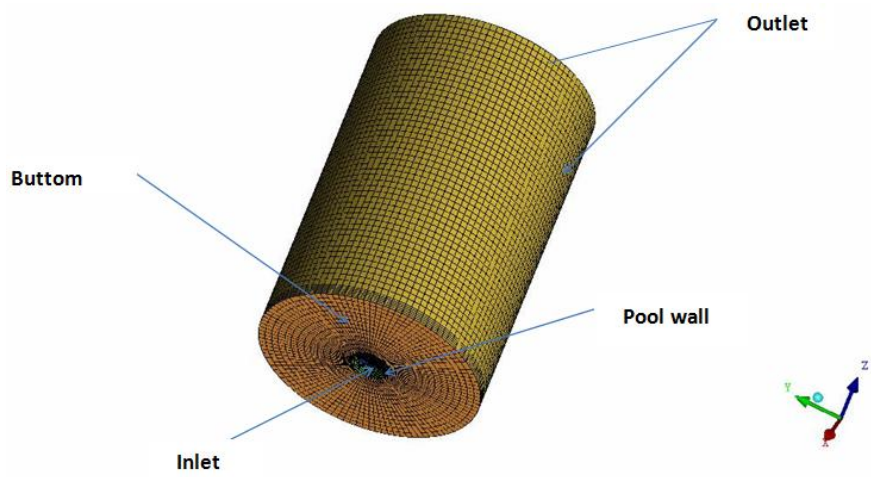

Fig 3: A scheme of the 3-D meshes applied in simulations

Altogether more than 400,000 control volumes are used for the solution of transport equations described before. Since the mesh-dependent studies are important for the numerical solution to be independent on mesh size, so it was made sure that the simulations are mesh independent. The different grid size and the number of the control volumes that had been used in this work is shown in "Table 2". The results of the prediction of axial time averaged flame temperature distribution using different grids for the pool fire of the kerosene in a pool diameter of $0.06 \mathrm{~m}$ is shown in "Figure 4". As can be seen the further refinement of the grids from grid-2 to the grid-3 have minor effect on the axial flame temperature distribution of the kerosene thus the sizes corresponds in grid3 is applied for the simulations carried out in this work.

Table 2

\begin{tabular}{lllll}
\hline case & $\begin{array}{l}\text { Max x } \\
\text { size(m) }\end{array}$ & $\begin{array}{l}\text { Max y } \\
\text { size(m) }\end{array}$ & $\begin{array}{l}\text { Max z } \\
\text { size(m) }\end{array}$ & $\begin{array}{l}\text { Number } \\
\text { of Grids }\end{array}$ \\
\hline Grid-1 & 0.02 & 0.02 & 0.03 & 182909 \\
Grid-2 & 0.02 & 0.02 & 0.015 & 210195 \\
Grid-3 & 0.01 & 0.01 & 0.015 & 315490 \\
\hline
\end{tabular}

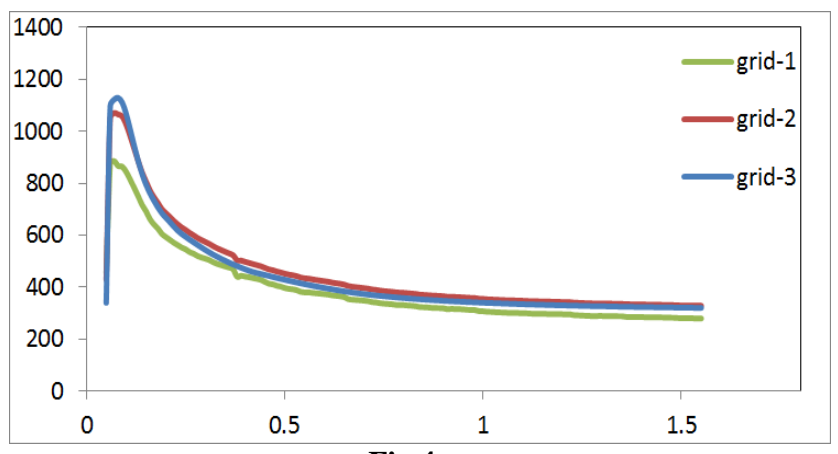

Fig 4

The fire domain initially contains air under ambient conditions: $\mathrm{T}=298 \mathrm{~K}$ and $\mathrm{P}=1.013$ bar. The definition of simplified geometry leads to a significant reduction of the time. As the fluid in domain an air is assumed to consist of nitrogen, oxygen and carbon dioxide. The existence of the species carbon dioxide and water at the beginning of the reaction when eddy dissipation model is used is essential. The mass fractions of individual species in temperature in domain is $\mathrm{T}=298 \mathrm{~K}$ and the pressure is defined relative to the reference pressure $\mathrm{P}=1.013$ bar. In the inlet boundary the fuel is assumed to be already evaporated and the fuel vapor coming from the inlet boundary surface with defined constant temperature and a constant mass flow rate of fuel equal to the mass burning rate, experimentally determined for different 
fuels. The inlet is surrounded with a low rim ("Figure 3") and adiabatic ground area. The remaining areas in the computational domain are open boundaries with defined ambient conditions. At the time $\mathrm{t}=0 \mathrm{~s}$, the flame is ignited in the experiment, or begins in the computational modeling. From that time initially grows in the size until sometime after its full size is reached. In the first test calculations have shown that the flames reach their full size after about $t=5 \mathrm{~s}$. In simulations the constant mass burning rate is used. For the pool fires simulations time steps in a range of $0.0001 \mathrm{~s} \leq \Delta \mathrm{t} \leq$ $0.001 \mathrm{~s}$ are chosen to reach achieved convergence level of minimum $10^{-3}$ independently on pool diameter but depending on the sub-models. Number of iterations per time step varied from 10 to 40 depending on chosen sub-models, where at the beginning of simulation a large number of $20-40$ iterations per time step is used and after the convergence is reached the number of iterations decreased to minimum 10-15.

\section{RESULT AND DISCUSSION}

\subsection{Flammable Material burning rate}

The dependence of mass burning rate of flammable material as a function of pool diameter for different materials is shown in "Figure 5". The mass burning rate of each of the materials is increased with an increase in diameter. The heat flux from the flame to the liquid surface increases with an increase in liquid surface area which results in an increase in flammable liquid evaporation rate in larger diameters.

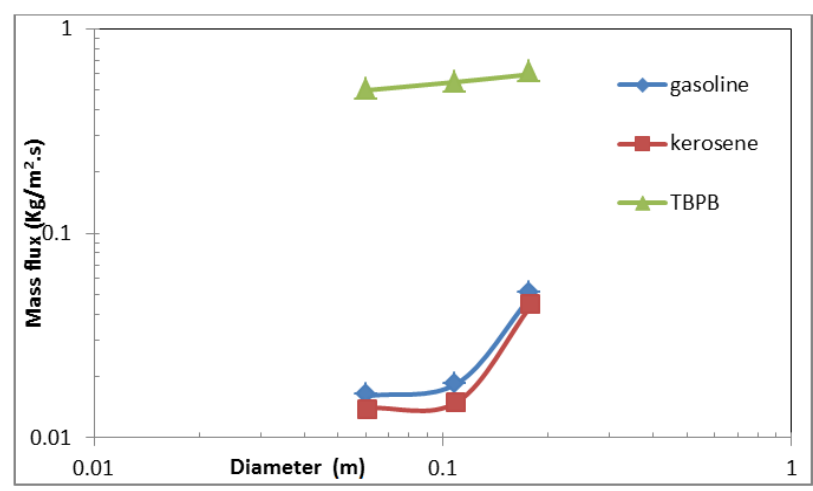

Fig 5: Experimental measured mass burning rates for gasoline, kerosene and TBPB

Also as can be seen organic peroxide TBPB burns almost 10 to 30 times of the common hydrocarbon fuels kerosene and gasoline. This is due to the basis of chemical structure of the fuel and the higher flame temperature of TBPB in comparison to the kerosene and gasoline as shown in "Table 3". which results in larger radiation heat flux to the liquid surface based on Stefan Boltzmann radiation heat law that describes the radiation heat flux as a 4.th power of the temperature.

Table 3

Experimental measured maximum flame temperature in different diameters.

\begin{tabular}{cccc}
\hline Diameter (m) & $\mathbf{0 . 0 6}$ & $\mathbf{0 . 1 1}$ & $\mathbf{0 . 1 8}$ \\
\cline { 2 - 4 } Kerosene & 1110 & Temperature $(\mathrm{K})$ & \\
Gasoline & 990 & 1331 & 1321 \\
TBPB & 1345 & 1569 & 1250 \\
& & & 1603 \\
\hline
\end{tabular}

\subsection{Flame height}

The experimental visible flame height of different flammable materials as a function of pool flame diameter is depicted in "Figure 6". As shown the visible flame height of each flammable material is increased with an increase in pool diameter which is due to the higher mass burning rates that extends flame visibility to a larger length. Also as can be seen the flame of TBPB extends about 2 to 4 times of the flame of kerosene and gasoline which is due to the 10 to 30 times larger burning rates of TBPB in comparison to the kerosene and gasoline as stated before. A schematic of the flame of each material is shown in "Figure 7".

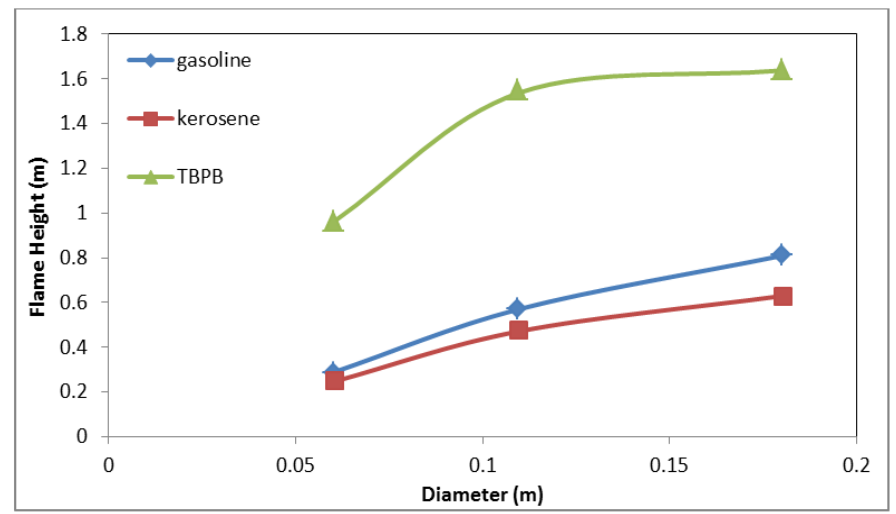

Fig 6

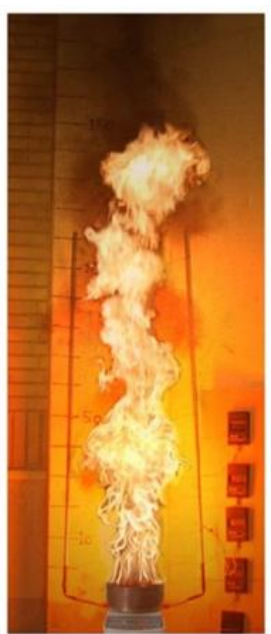

TBPB

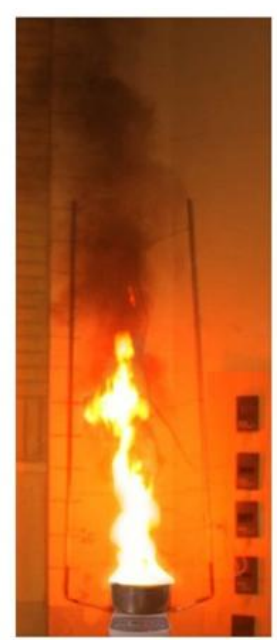

Gasoline

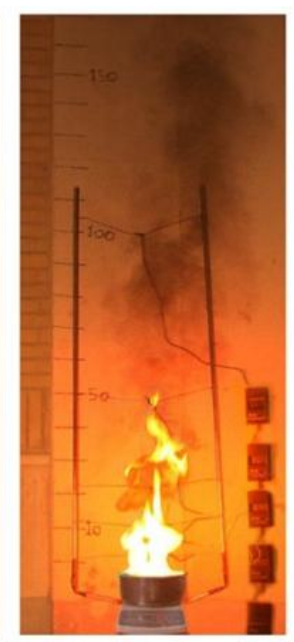

Kerosene
Fig 7

\subsection{Flame Temperature}

The CFD predicted instantaneous iso-volumes of flame temperature distribution for the simulations using RSM and LES turbulence models for a pool fire of $0.18 \mathrm{~m}$ diameter of different flammable materials is shown in "Figure 8". As can be seen in all of the simulations the three different zones of a fire is well captured. But as can be seen the high turbulence and intermittency in the plume zone is not well captured using RSM model while the turbulence in this zone is well captured using high physics LES turbulence model. 


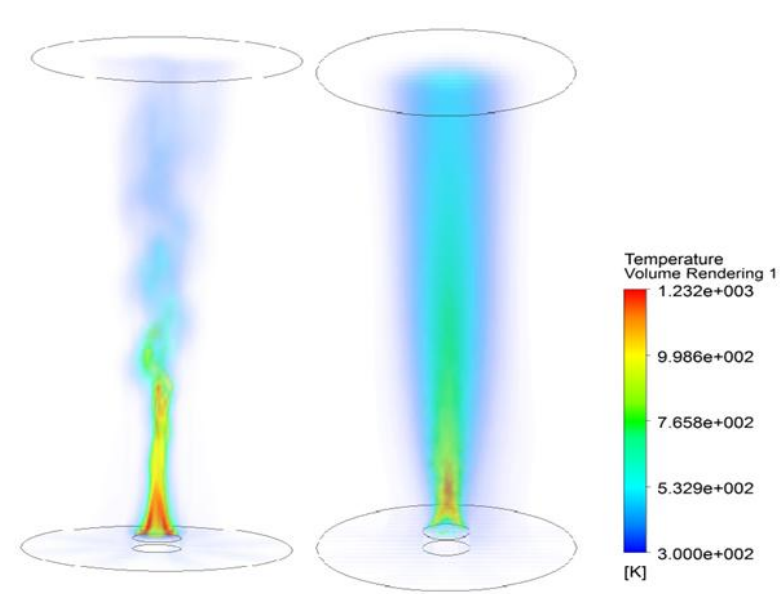

Fig 8.1:gasoline

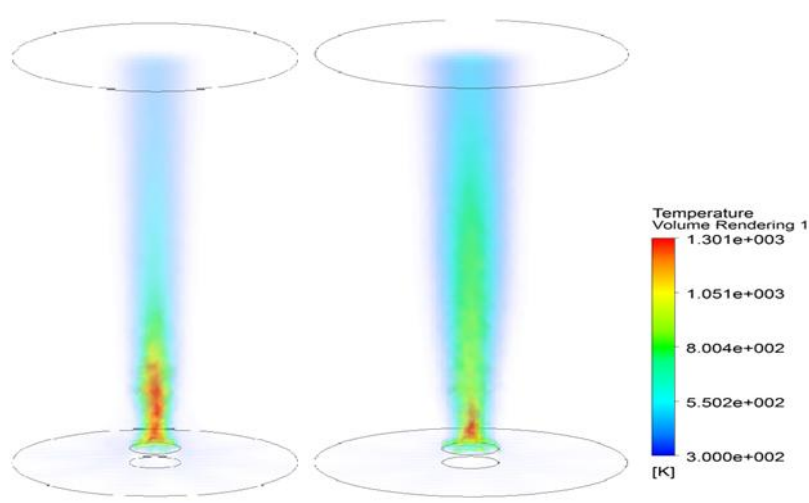

Fig8.2:kerosene

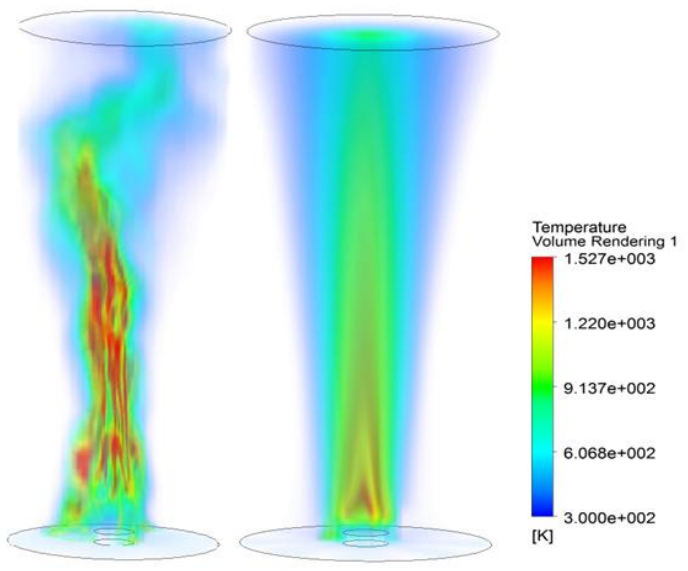

Fig 8.3:TBPB

The instantaneous flame temperature is monitored in some representative point in the simulation domain for each of the burning materials as indications of flow development and for simulation using LES turbulence model of a $0.18 \mathrm{~m}$ pool diameter of gasoline is shown in "Figure 9". As can be seen generally it takes about 5-6 seconds for each simulation to reach the quasi-steady state depending on the burning material and pool diameter. Once the fully developed, quasi-steady state is reached, the time-averaged quantities are calculated. To ensure the convergence of the averaged quantities the averaging processes are performed for 25 seconds.

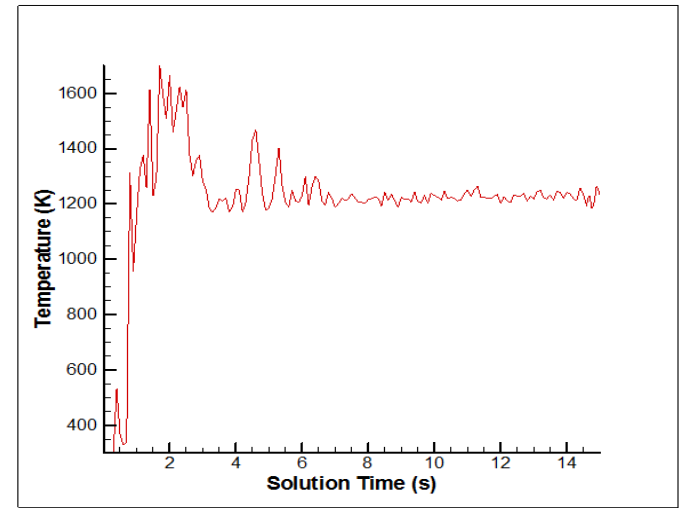

Fig 9

The experimental measured and CFD predicted time averaged axial flame temperature profiles for pool fire of each flammable material in different pool diameters is shown in "Figure 10". As can be seen in all the cases the trend of the experimental data is well captured using simulations although there is some quantitative discrepancy between CFD and experiments specially in the reaction zone which could be due to the low physics chemistry kinetic model which models the combustion reaction as a one-step reaction using a first order eddy-dissipation reaction model. Also as shown in "Figure 11 " the simulations using the LES turbulence model for the $0.18 \mathrm{~m}$ pool diameter is in much better agreement than the RSM turbulence model in "Figure 10.3" which is due to its better performance in modeling turbulence field that has an important impact on the combustion model predictions and flame flow field.

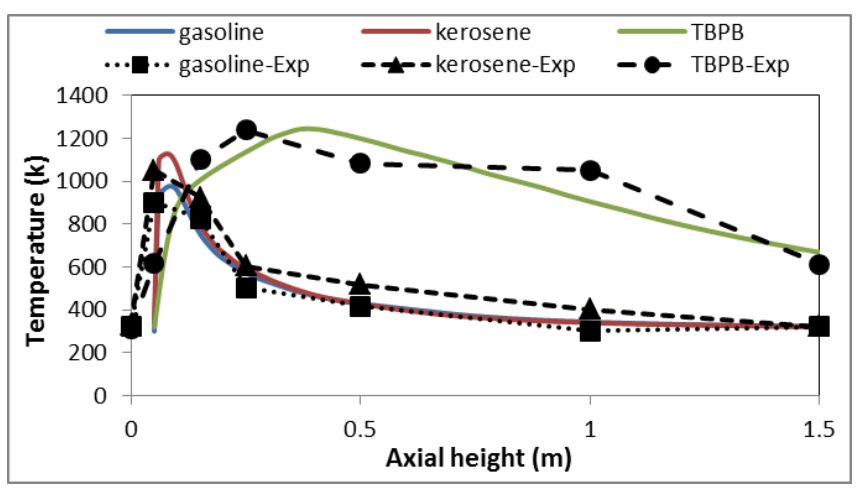

Fig 10.1:pool diameter 0.06m, RSM

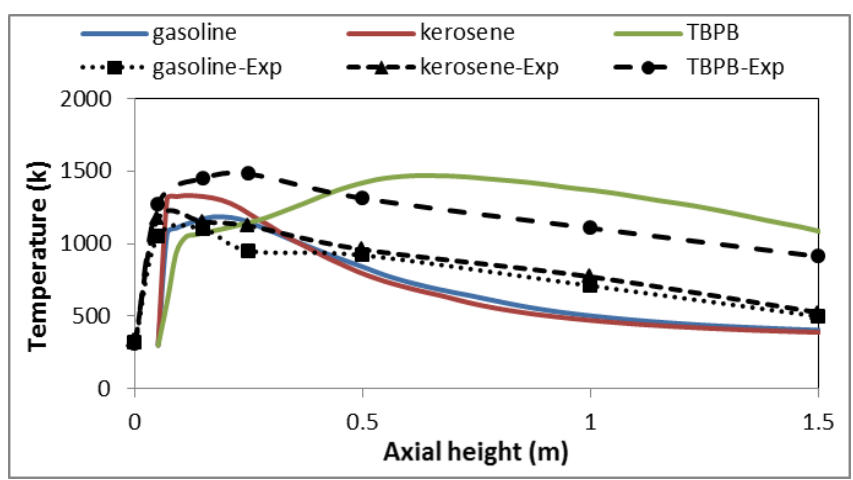

Fig 10.2:pool diameter 0.11m, RSM 


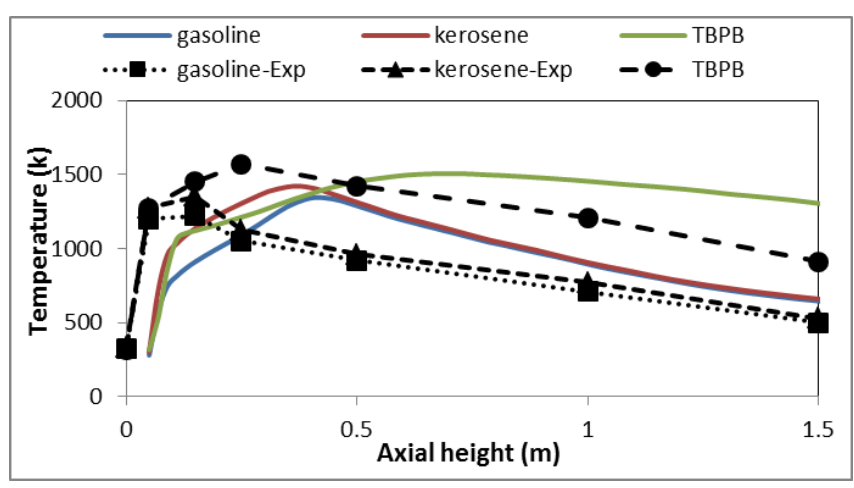

Fig 10.3:pool diameter 0.18m, RSM

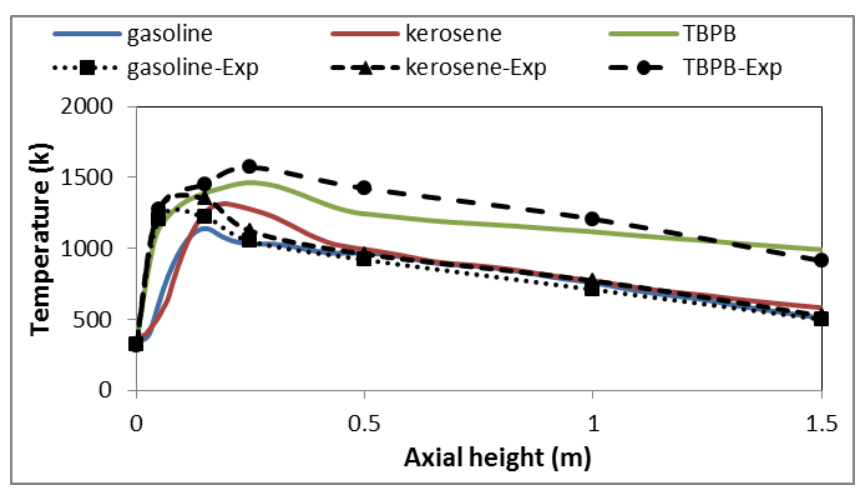

Fig 11:pool diameter 0.18m, LES

The CFD predicted flame development of $0.18 \mathrm{~m}$ diameter pool fire for different flammable materials which is studied in this work is monitored using iso-volumes of flame temperature in different solution times using LES turbulence model in "Figure 12". As can be seen the flame development of a typical peroxide (TBPB) due to its different chemical properties is about two times faster than the common hydrocarbon fuels gasoline and kerosene. This fact is important specially in the case of accidents and requires faster operations to controlling the fire.

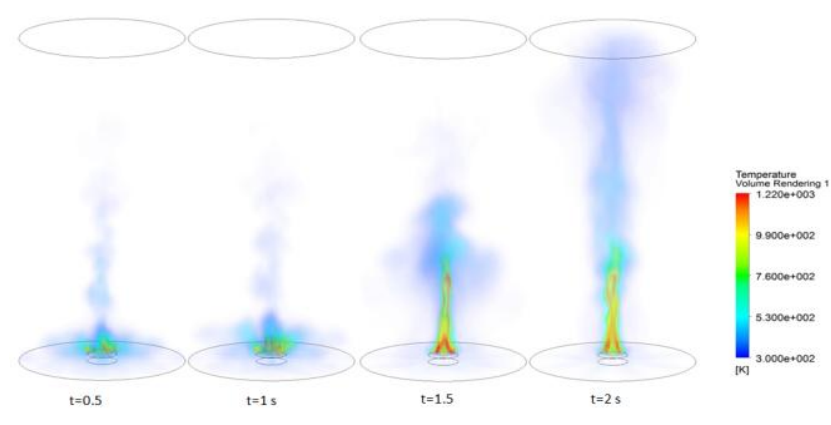

Fig 12.1:gasoline

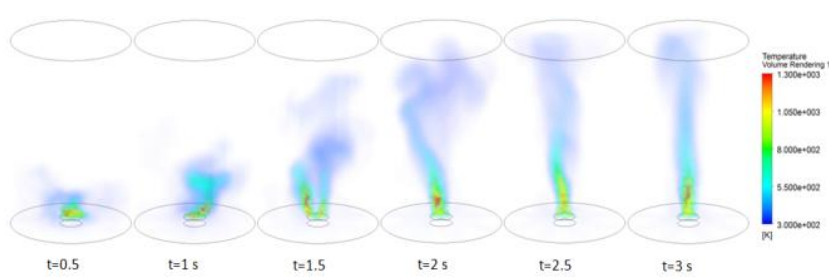

Fig 12.2:kerosene

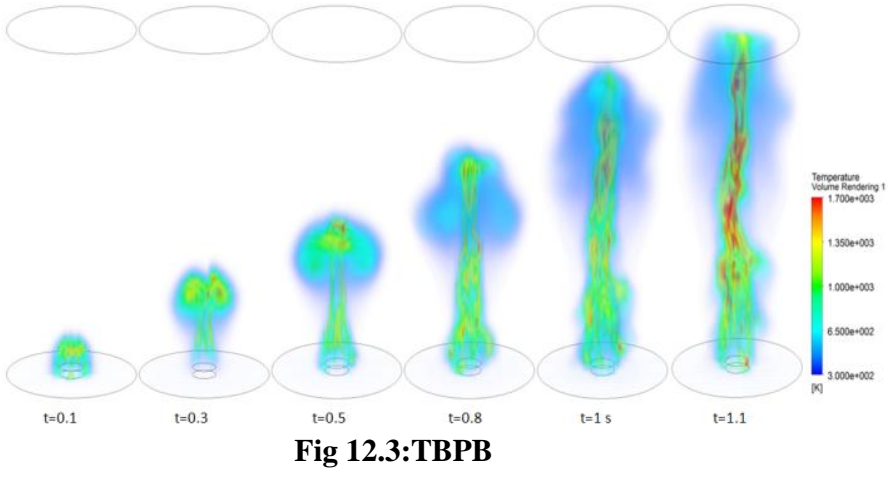

5.4 Flame irradiation

The flame irradiance $\mathrm{E}$ can be calculated by surface emissive power (SEP) of the flame using the following equations:

$$
\begin{aligned}
& E=\varphi_{F, R} \tau E_{F}^{S E P} \\
& E_{F}^{S E P}=\sigma \varepsilon_{F}\left(T_{F}^{4}-T_{a m b}^{4}\right)
\end{aligned}
$$

where the $\tau$ is the atmospheric transmissivity, $\varphi_{F, R}$ is the view factor from flame $(\mathrm{F})$ to receiver $(\mathrm{R})$ and is calculated using following equation [9]:

$\varphi_{F, R}=\frac{1}{2 \pi}\left(\frac{B}{\sqrt{1+B^{2}}} \arctan \left(\frac{C}{\sqrt{1+B^{2}}}\right)+\frac{C}{\sqrt{1+C^{2}}} \arctan \left(\frac{B}{\sqrt{1+C^{2}}}\right)\right)$

where $\mathrm{B}$ and $\mathrm{C}$ are defined as the ratio of flame width to distance between flame and receiver $(\Delta y)$ and flame height $(\mathrm{H})$ to distance between flame and receiver $(\Delta \mathrm{y})$ respectively for approximation of flame as a rectangular surface $[8,9] . \sigma$ is the Stefan Boltzmann constant $\left(5.67 \times 10^{-8} W /\left(m^{2} K^{4}\right)\right)$.

$\varepsilon_{F \text { is }}$ the flame emissivity which is calculated in this work using following procedure.Babrauskas (1983) [10] applied conservation of energy for the liquid surface as given below:

$\overline{\dot{m}}_{f}^{\prime \prime} \Delta h_{g}=\dot{q}_{r}^{\prime \prime}+\dot{q}_{c}^{\prime \prime}+\dot{q}_{r r}^{\prime \prime}+\dot{q}_{\text {loss }}^{\prime \prime} \quad$ (4)where $\overline{\bar{m}}_{f}^{\prime}$ is the mass loss rate per unit area (assumed identical to the burning rate), $\Delta h_{g}$ is the total heat of gasification, $\dot{q}_{r}^{\mathrm{m}}$ is the radiant flux absorbed by the pool, $\dot{q}_{c}^{m}$ is the heat received convectively, $\stackrel{\vec{q}}{r r}_{m}^{m}$ is the re-radiant heat loss due to the surface of the pool being at an elevated temperature and $\dot{q}_{\text {loss }}^{\text {Ioss is the lumped wall conduction losses and unsteady }}$ terms. Lumped wall conduction losses $\left(\dot{q}_{\text {loss }}^{\mathrm{m}}\right)$ and re-radiant heat losses $\left(\dot{q}_{r r}^{m}\right)$ are usually small and hence neglected. Thus the "(4)" can be simplified as :

$\overline{\dot{m}}_{f}^{\prime \prime} \Delta h_{g}=\dot{q}_{r}^{\prime \prime}+\dot{q}_{c}^{\prime \prime}$ 
Fay [1] calculated the convective heat flux from flame to liquid surface for circular pool fires as following relation:

$\dot{q}_{c}^{\prime \prime}=1.30 \times 10^{-3}\left(\rho_{\text {air }} \sqrt{g d}\left(\frac{-\Delta H_{c}}{1+f}\right)\right)(6)$

substitution of the radiation heat flux from Stefan-Boltzmann and convective heat flux from "(6)" into "(5)" and some mathematical manipulations results in following equation for flame emissivity $\varepsilon_{F}$ :

$$
\varepsilon_{F}=\frac{\overline{\dot{m}}_{f}^{\prime \prime} \Delta h_{v}-1.30 \times 10^{-3}\left(\rho_{\text {air }} \sqrt{g d}\left(\frac{-\Delta H_{c}}{1+f}\right)\right)}{\sigma T_{F}^{4}}
$$

The flame irradiances $E$ is computed using relation (1) with both experimental and CFD predicted maximum time averaged flame temperature in different horizontal distance $\Delta y$ from pool rim. The results plotted as a function of non dimensional $(\Delta y / d)$ distance from fire for each flammable material studied in this work and is shown in "Figure 13". As can be seen from the figures in all of the cases the CFD predicted results are in good agreement with experimental measured results. If one follows the criterion described in [11] i.e. NFPA (also given in "Figure 13") for designing the tanks and process equipment's is $5 \mathrm{~kW} / \mathrm{m}^{\wedge} 2$ based on the phenomenon of skin burn caused by radiant heat exposure whereas EN 1473 recommends this limit to be $1.5 \mathrm{~kW} / \mathrm{m}^{\wedge} 2$ $[11,12]$.This safety distances is calculated for both experimental measured and CFD predicted maximum time averaged flame temperature of each flammable material studied in this work in the case of pool fire of $0.18 \mathrm{~m}$ diameter and the results is reported in "Table 4" and "Table 5". Thus as shown this safety distance can be predicted with CFD with good accuracy.

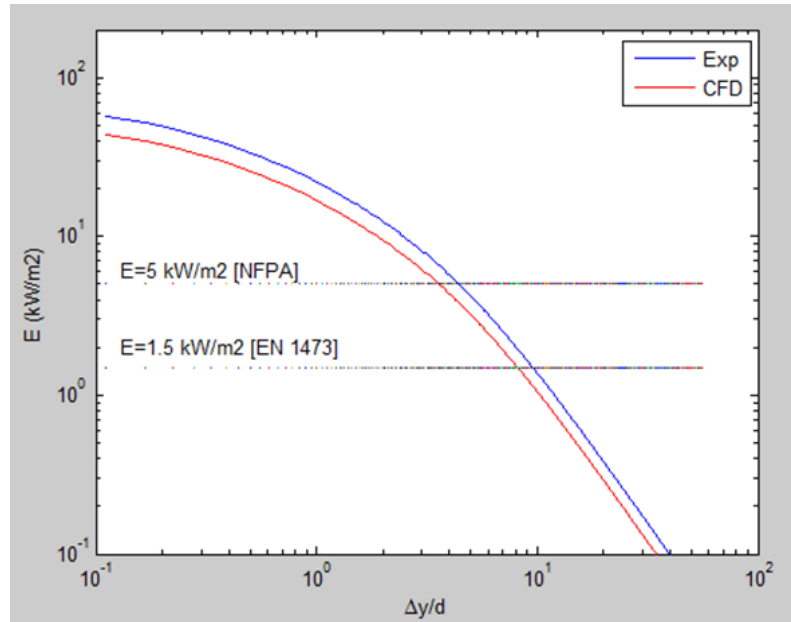

Fig 13.1:gasoline

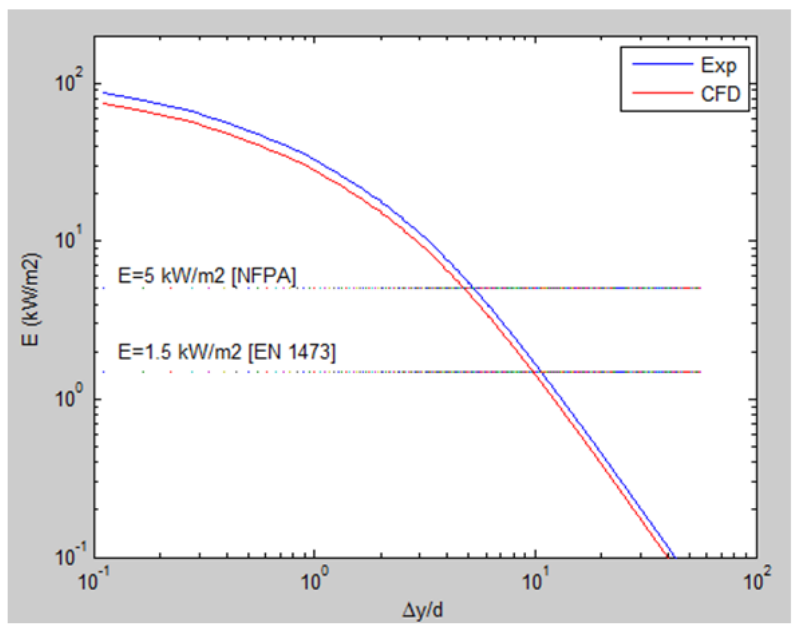

Fig 13.2:kerosene

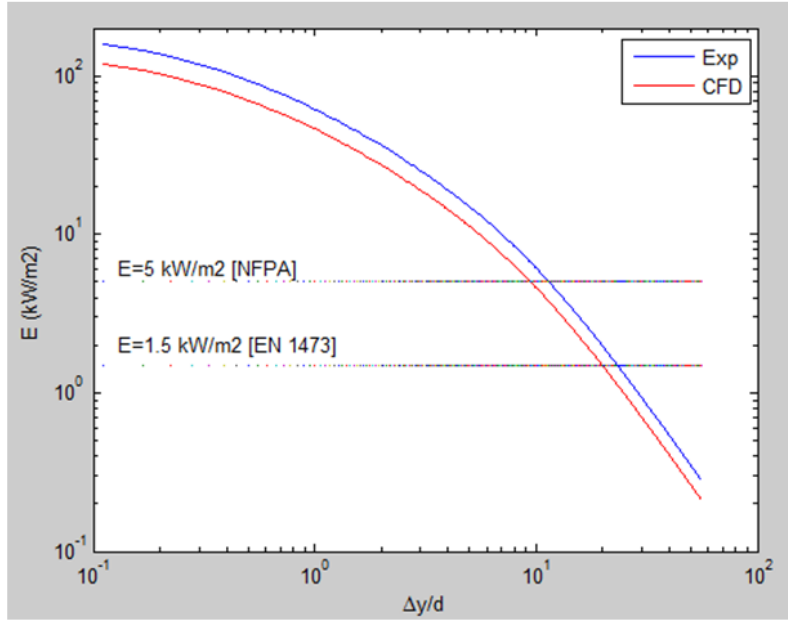

Fig 13.3:TBPB

Table 4

Experimental and CFD predicted safety distance corresponding to NFPA standard

\begin{tabular}{llll}
\hline & gasoline & kerosene & TBPB \\
\cline { 2 - 4 } Experimental(m) & 0.79 & 0.95 & 2.07 \\
CFD (m) & 0.6401 & 0.86 & 1.71 \\
Error (\%) & 18.9793 & 9.4733 & 17.3913 \\
\hline
\end{tabular}

Table 5

Experimental and CFD predicted safety distance corresponding to EN 1473 standard.

\begin{tabular}{llll}
\hline & gasoline & kerosene & TBPB \\
\cline { 2 - 4 } Experimental(m) & 1.73 & 1.9296 & 4.1706 \\
CFD (m) & 1.48 & 1.7694 & 3.5694 \\
Error (\%) & 14.4522 & 8.3022 & 14.4152 \\
\hline
\end{tabular}




\section{CONCLUSION}

In order to characterize and measure safety distances to protect personnel and equipment against the accidental encountered pool fires, a series of experiments and CFD simulations using different materials and different pool diameters is performed. To approach this aim pool fire of two hydrocarbon fuels (i.e. gasoline and kerosene) and a peroxide (TBPB (tert-butyl peroxybenzoate)) in different pool diameters (i.e. $0.06,0.11,0.18 \mathrm{~m}$ pan diameter) is studied. The results are as follows:

1- Experiments revealed that the pool fires of liquid organic peroxides show fundamentally very different characteristics e.g. generally much higher mass burning rates, large flame lengths as well as high temperatures and subsequently higher irradiances in comparison to liquid hydrocarbon pool fires. The higher temperature flame of TBPB in comparison to the gasoline and kerosene results in higher heat flux on to the liquid surface which results in higher vaporization rate of liquid pool and larger pool flames.

2- The three well-known flame zones of pool fires is well captured by CFD simulations and the predicted axial flame temperature profiles are quantitatively close to the measured values.

3- The CFD predictions using LES turbulence model in comparison to the RSM turbulence was in better agreement with experiments specially in the plume zone which is due to the higher physical accuracy of LES model and it's capability to model dispersion of large eddies in plume zone.

4- The CFD simulations showed that the pool flame of TBPB about two times faster develops in comparison to common hydrocarbon fuels gasoline and kerosene.

5- The safety distances accurately predicted by CFD simulations when predicted time averaged maximum flame temperature is used instead to the experimentally measured values in calculations performed in this work.

\section{ACKNOWLEDGMENTS}

This project is supported by consultancy funding generated in the research account under Areas of Strength in Fire Safety Engineering of the Petroleum University of Technology.

\section{REFERENCES}

[1] J.A. Fay, "Model of large pool fires", J. Hazard. Mater. 136 (2006) 219-232.
[2] Thomas PH, Baldwin R, Heselden AJM. "Buoyant diffusion flames: some measurements of air entrainment, heat transfer, and flame merging." 10th Symposium on Combustion, Cambridge, UK, 1965, pp. 983-996.

[3] McCaffrey B. "Purely buoyant diffusion flames-some experimental results.” NBSIR 79-1910, National Bureau of Standards, Washington, 1979

[4] Sinai YL, Owens MP. "Validation of CFD modeling of unconfined pool fires with crosswind: flame geometry." Fire Safety J 1995;24:1-34.

[5] B. J. McCaffrey; C. L. Beyler; G. Heskestad: "SFPE Handbook of Fire Protection Engineering", Third Edition, 2002, ISBN: 087765-451-4.

[6] T. Steinhaus; S. Welch; R. O. Carvel; J. L. Torero: "Large-scale pool fires", Journal of Thermal Science (2007)101-116.

[7] P. K. Raj: "Large LNG fire thermal radiation", AIChE Spring Meeting April 13(2005)219-232.

[8] P. Joulain: "Behaviour of pool fires: state of the Art and new insights, Proceedings", 27th Int. Combustion Symposium, (1999)2691-2706.

[9] A. Schonbucher; B. Arnold; K. Banhardt; V. Bieller; H. Kasper; M. Kaufman; R. Lucas; N. Scheiß: , 21 Symp.(Intl.) on Combust., The Combustion Institute 1986.

[10] Babrauskas, V., 1983. "Estimating large pool fireburning rates". FireTechnology 19, 251-261.

[11] P. K. Raj: "A review of the criteria for people exposure to radiant heat fluxes fromfires", Journal of HazardousMaterials 159(2008)61-71.

[12] EN 1473, European Standard on the Installation and Equipment for Liquefied Natural GasDesign of On-shore Installations, prEN 1473:2005(E), Standard Version 2.1c2, Prepared by the TechnicalCommittee CEN/TC 282, AFNOR Secretariat, Brussels. See Installation and equipment for LNG, Table A.2, p 83, The Secretariat of whichisheld by AFNOR, 1 September 2005. 